\title{
Technology-Augmented Choice: How Digital Innovations Are Transforming Consumer Decision Processes
}

\begin{abstract}
This paper provides an overview of recent research that explores how digital technologies such as mobile devices, wearables, voice technology, and recommendation agents are transforming consumer decision-making. We advance a conceptual model of technologyaugmented choice that describes how the three Ms of technology-mediums (i.e., device types), modalities (i.e., interaction interfaces), and modifiers (i.e., intelligent agents)—are becoming increasingly integral elements of consumer decision processes. For instance, today's new technologies often help curate consideration sets, shape how options are evaluated, and even guide choices themselves. As a result, market choices must now be viewed as a joint function of both consumer preferences and the characteristics of the technological environment in which those preferences are expressed. Examples of empirical research are reviewed that characterize the interdependencies between technology and decision-making, including how smartphones transform user-generated content, voice technology affects consumer search, haptic interfaces shape product preferences, and search engines alter confidence in choice.
\end{abstract}

Keywords: Artificial Intelligence, Smart Objects, Wearables, Voice Technology, Search Engines, Mobile Devices, Chatbots, Human Augmentation. 


\section{Introduction}

Recent advances in technology are reshaping how, when, and where consumers make choices. For example, $81 \%$ of U.S. adults now own a smartphone [67] and increasingly rely on these devices not just for mobile communication but also for shopping, banking, information gathering, and entertainment. Emerging technologies are being designed to further augment and automate many of the behaviors and decisions traditionally reserved for human decision makers. More than 20 billion "smart" interfaces such as Amazon Echo devices, chatbots, and sensors are connected to the Internet, already dwarfing the 7.35 billion people on the planet [41]. As these technologies continue to connect both with humans and with one other, they are increasingly transforming how consumers think, interact, and decide.

The purpose of this paper is to explore the nature of this transformation and its implications for firms and consumers. Our central thesis is that new technologies have done more than simply expand the variety of platforms through which consumers can access information - these technologies are also becoming an integral part of the consumer decisionmaking process itself, intelligently guiding, constricting, and in some cases, supplanting consumer preferences. As such, whereas market choices might have once arisen from a simple process in which consumers maximized their preferences over a set of options, today that process is far more complex, with primitive choice elements such as preferences increasingly intertwined with the medium through which choices are made (e.g., smartphones), the modes of interaction (e.g., haptics), and modifiers arising from intelligent software (e.g., recommendation agents). We argue that this shift calls for a reconceptualization of how researchers think about and study consumer decision-making. 
In this paper we provide an overview of this changing landscape in consumer behavior. Like any review, our discussion of new technologies and their impact is necessarily limited in scope. We omit from our discussion, for example, emerging work on the economic impacts of artificial intelligence [1], developments in online advertising [72], and philosophical and ethical issues in the design of AI $[61,98]$. Instead, we focus on emerging work on the psychology of new technologies—how interactions with new mediums (devices), modalities (interfaces), and modifiers (intelligent agents) affect the way consumers think, feel, and make choices.

We organize our discussion in three parts. We begin by providing an overview of research in this area and proposing a conceptual model that describes the ways in which technologies are changing traditional theories of choice. We then illustrate the model by reviewing findings from three major streams of research: how decision-making is being altered by new devices (smartphones and wearables), modalities (haptics, voice, and immersive visual interfaces), and the intelligent agents that often operate through these modalities (recommendation systems and search aids).

\section{How Technology Is Changing Choice}

Classic economic theories of choice give a straightforward account of the relationship that exists between the products offered by sellers and market choices. In the classic view, consumers are assumed to hold preferences over an array of choice options and to ultimately choose the alternative that maximizes these preferences [56]. Hence, there is an implicit correspondence between market choice and preference: if a consumer chooses widget A over widget $\mathrm{B}$, she or he must possess a preference ordering for widgets in which $\mathrm{A}>\mathrm{B}$.

We argue that the widespread adoption of technological innovations that aid—and, in some cases, supplant—thoughtful choices requires a revision of this classic view. Of course, the 
notion that choice can be influenced by the technology that facilitates it is by no means new. The introduction of radio, television, and the Internet, for example, greatly expanded the number of alternatives from which consumers could form their consideration sets and widened the number of channels through which choices could be made [18]. Indeed, because preferences are often constructed in response to the particular choice context $[4,86]$, one could argue that these past technological advances had no less of an impact on decision making than new technologies have today.

We contend, however, that while today's new technologies similarly expand the range of alternatives and channels available to consumers, they differ from past innovations in a number of notable ways. For one, new technologies are having novel effects on choice, through the introduction of new mediums (e.g., smartphones, wearable devices) as well as new modalities of interaction (e.g., haptics, voice) that can alter the emotional and cognitive bases of decisions. Further, today's new technologies are unique in their ability to intelligently guide choice by offering personalized information to consumers.

Consider, for example, a consumer using her smartphone to choose a product to purchase on Amazon. In some ways, this choice is similar to those considered in the classic paradigm: the consumer is presented with a set of options and chooses the one that she or he most prefers. However, there are also key differences that cannot be overlooked. For one, engaging with new mediums such as smartphones has been shown to alter consumers' situational feelings during decision-making [22] relative to the use of older technologies such as personal computers [59, 58]. These new mediums also involve new modalities such as touch and voice; in this example, the haptic interface of smartphones can provide vibro-tactical feedback that directs which cues consumers attend to in their environment [32]. In addition, today's new technologies often 
involve intelligent modifiers of choice, such as the recommendation system underlying Amazon's platform. These intelligent modifiers allow platforms such as Amazon to not only distribute a set of options from which consumers choose but also to act as intelligent curators, giving the consumer a set of options that their algorithms predict will be most preferred by him or her [3]. As a result, new technologies can serve as an important partner in decision-making, with choice being a joint function of the consumer's preferences and the modifier's algorithmic representation of those preferences.

We contend that as consumption activities continue to play out in more advanced technological environments, it will become increasingly difficult to parse out whether choices made in the marketplace are driven by consumers' preexisting preferences or preferences shaped by the technological context - the mediums, modalities, and modifiers - in which they make these decisions. As a result, the technology-augmented choices made by consumers today are rendering the traditional direct relation between preference and choice virtually obsolete.

\section{Technology-Augmented Choice: A Conceptual Framework}

Figure 1 presents a conceptual model that we refer to as the " 3 Ms of technologyaugmented choice," characterizing the various interdependencies that exist among features of technology, consumer preference, and market choices. The model posits that market choices are increasingly the consequence of an interplay between primitive choice elements (i.e., consumers' latent preferences, emotions, and/or knowledge) and at least one of three features of the technological environment, namely: 1) the medium, or device type (e.g., wearables, smartphone), 2) the modality, or interaction interface (e.g., haptic, voice), and/or 3) the presence of modifiers, or intelligent choice agents (e.g., recommendation systems, search engines). Taken together, such technological features form a decision-making ecosystem in which technology is no longer 
merely a passive facilitator of choice but plays an active role in guiding, altering, and in some cases making consumer choices.

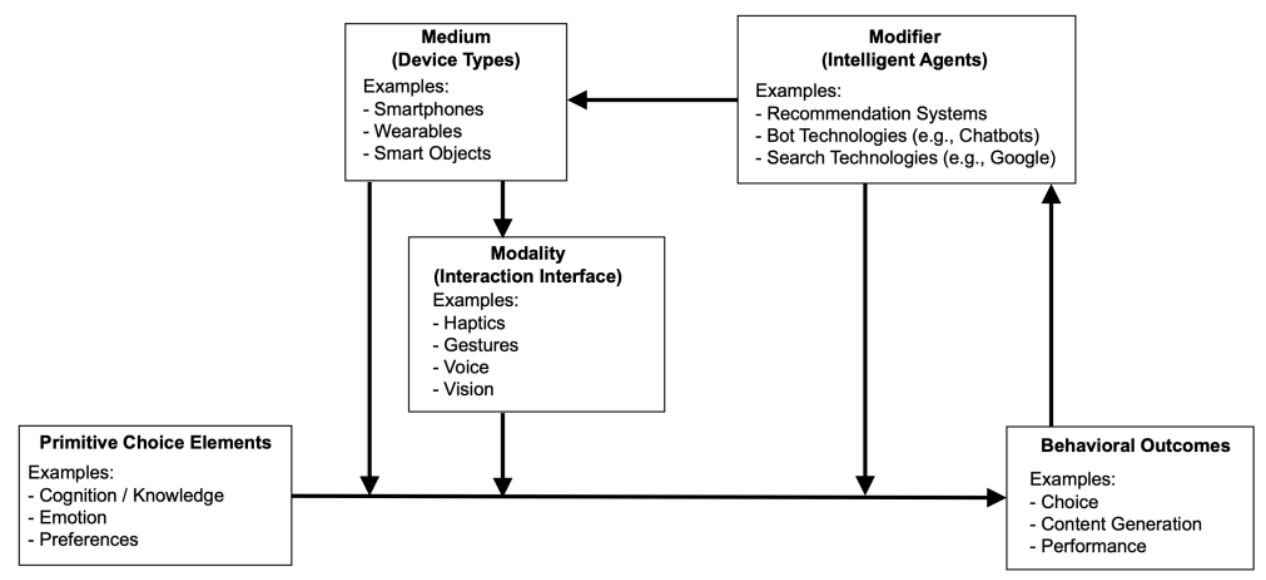

Figure 1: The 3 Ms Conceptual Model of Technology-Augmented Choice

The model displays several properties that have relevant empirical implications for the study of choice. For one, the model posits that in some cases consumer preferences themselves could be altered by the particular digital platform with which one engages - for example, whether one is making a choice on a smartphone versus laptop [58], or whether actions taken on a device are controlled through voice versus keyboard/mouse [50]. Likewise, the model implies that choices and preferences are at least partially endogenous. When searching with the aid of a recommendation system, for example, consumers are presented with the agent's predictions of what the consumer wants to see-which is partly a function of what that consumer has chosen in the past. This recursivity becomes even more complex in the case of smart objects, where representations of consumers' preferences reflect not just their past behaviors but possibly the machine's past behaviors as well [40]. For example, Roomba robotic vacuum cleaners are programmed to learn from their own past mistakes in encountering obstacles [5]. 
Below we offer a review of some of the recent findings on how choice processes are uniquely influenced by each of these three technological features (medium, modality, and modifier). We first discuss work investigating the choice-related effects associated with two mediums at the forefront of technology-augmented choice - smartphones and wearables. We then discuss the modalities these devices tend to involve-namely, haptics, voice, and immersive visual interfaces. Finally, we review work on the effects of the most well-known modifiers of choice: intelligent agents and virtual decision aids, specifically recommendation systems and search engines.

\section{The Medium: Effects of Device Types on Choice}

Perhaps the most visible new technologies are the novel mediums on which consumers are browsing, gathering information, and making decisions - two pervasive examples being smartphones and wearable devices. What has made these technologies particularly transformative is not just the expanded functionalities they afford but their near-constant presence in consumers' everyday lives, with smartphones and wearables virtually always on one's person. As discussed below, an important implication of the close "pairing" between consumers and their new devices is that instead of merely providing an additional platform for choice, these new mediums can actually alter users' psychological experiences during choice processes and thereby shape the choices themselves.

\subsection{Smartphone Use and Its Consequences}

The proliferation of the smartphone over the past decade has changed the way consumers browse and consume products. More profoundly, the device has also transformed how users think and communicate. The portability of smartphones affords users near-constant access to the wide array of functionalities available on the device (e.g., informational, entertainment, and 
communication features). Perhaps unsurprisingly, the majority of owners now see their smartphone as a device they cannot live without—an attachment that, for some, can resemble an addiction [64]. As a burgeoning body of research illustrates, the use of these new constant companions can have meaningful effects on cognition, emotion, and communication.

One consequence of the strong bond that many consumers seem to form with their smartphone is a reliance on the device as a source of psychological comfort in moments of stress. In a series of lab and field studies, Melumad and Pham [59] find that engagement with one's smartphone enhances users' feelings of psychological comfort not unlike those provided by childhood attachment objects (e.g., security blankets). As an illustration, when lab participants underwent a stress induction, they disproportionately reached for their smartphones for solace over other objects at their disposal, and doing so helped ameliorate their level of stress. Moreover, this effect was unique to one's smartphone per se: comparable reductions in stress were not observed when participants engaged in the same task on their laptop or even with a similar smartphone belonging to someone else. The authors show that this "special" relationship to one's phone (relative to other devices) arises from its unique combination of properties: its portability, highly personal nature, the sense of privacy experienced while on the device, and the haptic benefits it offers.

Moreover, this strong bond with one's smartphone can yield psychological effects even when the device is not in use. For example, growing evidence suggests that unattended notifications from one's phone often induce mind-wandering and can thus undermine performance even when the device is not in use $[51,79]$. It is perhaps more intriguing to note that the mere presence of one's own smartphone may reduce available cognitive capacity even when it does not actively grab attention by ringing or buzzing. As a test of this possibility, Ward 
et al. [92] had participants complete a series of cognitive tests after placing their smartphone in one of three locations: on the desk in front of them, in their pocket or bag, or in a separate room. All participants were successful at avoiding the urge to check their phones. However, the results showed that cognitive performance declined as proximity to one's phone increased, and this effect was greatest for those who used their phones the most—-suggesting that resisting the urge to attend to one's phone requires cognitive effort. Relatedly, additional work has shown that based on both self-report and physiological measures, people experience heightened anxiety when separated from their smartphone $[15,17]$.

An important implication of these findings is the possibility that the choices made on one's phone may differ from those made on other devices. For example, prior work on the effects of mood on decision-making $[43,62]$ suggests that, to the extent that engagement with one's smartphone is a source of positive affect, consumers making choices on their smartphones may be more prone to seek variety [47], be more open to considering innovations [43], and be more likely to assume risk [78].

Smartphone use has also been shown to alter consumer behavior in the domain of usergenerated content. Research by Melumad, Inman, and Pham [57] and Ransbotham et al. [70], for example, finds that user-generated content (such as restaurant reviews and social media posts) written on smartphones tends to be more emotional than that written on personal computers. While the mechanisms that lead to these effects are presumably diverse, the physical properties of the device appear to play a significant role. For example, Melumad et al. [57] show that the greater observed emotionality of user-generated content produced on smartphones is driven, at least in part, by the relatively small size of the keyboard and screen, which constrains users to convey the gist of their experience. That is, rather than conveying more nuanced details, 
consumers generating reviews on their smartphone tend to selectively include their affective reactions to an experience, resulting in content that appears more emotional to readers.

Other work by Melumad and Meyer [58] finds that generating certain types of content on one's smartphone (vs. PC) can lead consumers to exhibit enhanced self-disclosure, sharing feelings and thoughts that are more intimate or private. The authors find that this effect arises from both the greater psychological comfort experienced on the device (consistent with the findings of Melumad and Pham [59]) and greater focus on the task at hand due to its smaller screen. In particular, prior work has documented that the small size of smartphone screens leads users to experience "attentional blindness" when on their device, leading them to become deeply engrossed in the activities they engage in and in turn rendering them oblivious to external distractions $[42,53]$. As such, smartphone users tend to direct their cognitive focus to the task onscreen —in this case, the disclosure at hand — which leads them to focus more on their feelings and thoughts related to that disclosure (rather than distractors that might otherwise suppress tendencies to self-disclose). These findings can help explain one apparent paradox of smartphone use: that consumers often engage in highly sensitive personal activities in very public settings (e.g., writing a private text to a friend while on a crowded subway).

\subsection{The Effects of Wearable Technology}

Wearable technologies— such as smart watches, fitness trackers, and medical wearables - represent the next step toward integrating technology into the minutiae of everyday life. Whereas smartphones place the benefits of technology in our pocket, wearables place these benefits on our person. In contrast to research on smartphones, however, research on the effects of wearable technologies on decision-making is in an even more nascent stage [16]. Most of the extant work discusses wearable technologies in the context of their ability to offer biological 
feedback (e.g., heartrate, sleep quality, steps, glucose monitoring) that, in principle, could allow users to make better decisions for achieving their health goals. While this is most often portrayed as a benefit to individual users (e.g., letting users know whether they achieved a good night's sleep), the information provided by wearable devices can also offer community-level benefits when analyzed by health-care professionals. For example, Radin et al. [69] recently illustrated that influenza outbreaks can be mapped by analyzing the geographical distribution of heartrate and other markers by Fitbit users.

There is also some accumulating evidence, however, that providing users with an ability to obtain nearly continuous measurement of health or other states may have unintended negative side effects. For example, Jakicic et al. [44] reported the findings of a clinical trial in which participants were randomly assigned to receive a Fitbit-like device (vs. not) to monitor exercise activity as part of a two-year weight-loss program. Rather than aiding in weight loss, use of the device seemed to hurt it, as participants in the control group actually lost more weight than those who used the Fitbit-like device.

While the reason for this result was unknown, one possible explanation might be drawn from the work of Etkin [26], who explored the effect of quantifying otherwise pleasurable activities (e.g., walking, reading) on the amount that these activities were engaged in and how much they were enjoyed. The primary finding was that quantification-e.g., being reminded of how many steps one took via a pedometer-is a doubled-edged sword: on one hand it increased the quantity of an activity that was engaged in, but it also decreased the level of reported enjoyment. This occurred because the focus on quantification drew users' attention away from the aesthetic aspects of an activity that would have otherwise contributed to its enjoyment. As such, it is possible that using wearable technology to quantify progress in a weight-loss program 
might cause participants to frame the benefits of the program more in terms of its extrinsic rather than intrinsic rewards, a motive that is known to be less effective in achieving health outcomes [52].

There is also emerging evidence that wearable technologies are prone to their own form of "dependence," specifically to the quantifications they provide. For example, an individual might feel unable to work out if the battery on her smartwatch (that measures activity) dies, or that a walk was "wasted" if her Fitbit failed to measure her steps [9]. Thus, while reliance on a smartwatch for notifications can help wean users from their dependence on their smartphone [20], this might actually just be shifting attachment to another personal device.

Now that we have discussed two transformative new devices that are changing consumer decisions and outcomes, we next describe the effects of some of the new interaction modalities available on these devices: haptic, voice, and visual interfaces.

\section{The Modality: Effects of New Modalities on Search and Choice}

One of the central features of the conceptual model in Figure 1 is the idea that consumer preferences - and, in turn, choice outcomes — can be affected by the particular modality through which consumers interact with the device. Touchscreens have become the dominant interface for mobile devices, voice-operated smart objects embed digital capabilities into our tangible world, and immersive visual technology allows us to virtually travel beyond our current physical environment. These trends have cumulatively added multisensory richness to digital experiences, and a burgeoning research stream has begun to explore how such shifts might influence consumer choices.

\subsection{Haptics}


Consumer engagement with technology has grown increasingly tactile over the years. One example of this is an experimental study by Brasel and Gips [7], who found that when laboratory participants chose a hotel room on a computing device using a touchscreen versus a mouse, they focused more on the tangible attributes of rooms (e.g., sheets and décor). This effect was consistent with a transference process in which hand actions selectively activated congruent semantic elements in memory. In particular, the act of touching room elements on a screen enhanced the salience of the tangible attributes of rooms when making a choice. Similar effects in the context of online food preferences have been reported by Shen et al. [75], who found that ordering food through a touchscreen on a tablet enhanced preference for hedonic or affect-laden options compared to ordering by using a computer mouse. Relatedly, Hildebrand and Levav [39] report that using a greater range of easy-to-perform gestures on touch-enabled devices increases perceptions of playfulness and leads to a greater tendency to add more features to custom-made products, and Mulcahy and Riedel [63] find that adding haptic features to in-store ad displays increases their effectiveness.

While the aforementioned research demonstrated consequential responses to the haptic properties of devices touched by consumers (with the device acting as a passive agent), other research examines consumer responses to haptic exchanges "initiated" by the device itself (with the device acting as an active agent). Namely, some scholars have begun to explore how haptic feedback (e.g., vibrations emitted by a device) can affect consumer preferences and choices. One example is work by Hampton and Hildebrand [34], who studied the behavioral impact of vibrotactile feedback on consumer choice. They found that shoppers on an online groceryshopping website who were randomly assigned to experience a vibration when hitting the "add item to cart" button included more items in their basket than those who experienced no vibration. 
Other recent work by Hadi and Valenzuela [32] demonstrates that haptic feedback accompanying message content can even improve consumers' task performance. In a series of lab and field studies, users received motivational messages from a virtual coach (e.g., encouraging them to be more physically active) on their mobile phone or smartwatch. Participants who received messages paired with haptic alerts did better on related tasks (e.g., achieved more steps) than those who received the same messages without haptic feedback. This effect manifested because haptic feedback increased feelings of the coach's social presence, in what otherwise felt like an impersonal technological exchange.

There is also evidence, however, that the effects of vibrotactile feedback may not be universally positive. Hadi et al. [33] studied how augmenting mobile ads with vibrations that dramatize action might affect consumer responses to brands. Drawing on prior work showing that haptic feedback from a person (e.g., interpersonal touch) has a positive effect on attitudes only when the receiver has feelings of warmth toward the initiator of the touch [65], Hadi et al. [33] hypothesized that haptic augmentation would boost attitudes only toward brands with which consumers associate analogous feelings of warmth. Consistent with this, consumers had more positive reactions to haptic augmentation in ads for warm brands (e.g., Cadbury) compared to unfamiliar or cold brands (e.g., Peugeot).

Finally, it is also compelling to consider how brands might take advantage of such capabilities. For example, Visa recently created a haptic "signature" to accompany point-of-sale transactions completed with a mobile phone or smartwatch [95]. Just as brands have historically developed proprietary visual logos and auditory jingles, it is intriguing to consider how they might take advantage of the "tactile toolbox" inside mobile phones and wearables to create tactile signatures. Future research can examine not only how and whether haptic signatures might 
improve branding efforts, but also explore what specific haptic sensations map onto brand associations.

\subsection{Voice Technology}

The above discussion touches upon the novel effects of tactile and vibrotactile modalities on consumer decision-making. In addition to this, recent advances in natural language processing have given rise to other new modalities of consumer-technology interactions, such as voiceenabled technology (e.g., Apple's Siri or Amazon's Alexa). The use of such voice-controlled modalities is having profound effects on how humans search, shop, and express their preferences. Evidence from the information and computer science literature suggests that voicebased (vs. text-based) search is performed fundamentally differently; for example, work by Jeng et al. [45] revealed that despite speech being the natural mode of human expression, voice-based search queries are perceived as more difficult to perform compared to text-based search queries. Additional research showed that voice-based search resulted in a greater number of search failures, which in turn caused consumers to systematically adjust the syntactical nature of a query - for example, by substituting more complex words and sentence structures for simpler ones, and even by removing words altogether [46]. These new affordances of voice-based technologies also highlight the potential to experience frustration during such interfaces. In recent work, Hildebrand, Efthymiou, and Busquet [38] provide evidence that certain emotional states that arise when using a smart speaker can be reliably predicted based on the user's vocal features. For example, their findings illustrate that feelings of frustration after repeated failure during a "conversation" with a digital voice assistant are encoded during speech formation via an increase in loudness (i.e., greater amplitude of a soundwave) and pitch (i.e., a higher frequency 
of a soundwave), along with variability in the vocal characteristics of a human speaker (i.e., higher entropy or "jitter" of a soundwave).

Services like Amazon Polly and Google Dialogflow further highlight the new ways in which interfaces are being designed to be highly personalized and adaptive, such as by altering the vocal characteristics of a digital voice assistant through speech synthesis (i.e., the production of auditory output by a machine based on predefined characteristics such as frequency, amplitude, vocal breaks, or other vocal characteristics [85]). For example, recent work by Braun et al. [8] on personalized in-car speech interfaces revealed that such synthesized digital voice assistants_—which, in this case, mimicked the speakers' own vocal characteristics — were perceived as more trustworthy, likable, and useful when compared to unmatched in-car voice assistants.

\subsection{Visual Technology}

Unlike haptics and conversational agents, the attempt to augment consumer decisions with immersive visual interfaces has a long history in marketing $[2,12]$. In prior work a distinction is made between applications that utilize virtual reality, in which 360-degree immersions are created, and applications that utilize augmented reality, in which interactive features are overlaid on a two-dimensional device (e.g., allowing users to rotate images of products on their smartphone screens $[2,76]$. Recent years have brought rapid growth in the development of both of these technologies, with applications ranging from computer gaming [74] to retailing $[2,29]$.

When first developed in the 1990s, such visualization tools were seen in marketing as a means for researchers to study consumer behavior in simulations that mirrored actual shopping environments. Illustrative of such efforts were "virtual store shelves" developed by researchers 
who found that choice behavior in augmented-reality environments often mirrored that observed in actual shopping settings $[12,13]$. Likewise, Urban et al. [87] utilized virtual reality in the design of "information accelerators" that allowed consumers to experience the process of learning about new products that have yet to be launched in the marketplace. More recently, this same technology has been used for such diverse applications as forecasting demand for space tourism [73] and even as a means of allowing homeowners to simulate what it is like to ride out a hurricane disaster in their home [60].

Beginning in the early 2000s, such visualization technologies became increasingly seen as more than a research tool and were recognized for their potential to serve as a means of enhancing online shopping experiences. Perhaps the most ambitious of these efforts was Linden Lab's "Second Life," a PC-based massive multiplayer simulation that allowed users to play the role of virtual consumers who could live, work, and buy actual products from real companies. Seeing this as a potential new channel of commerce, a large number of firms - including Adidas, American Apparel, and Dell—opened virtual stores in the simulation [48]. But after a rapid surge of popularity, Second Life lost its appeal, and virtual stores closed down by 2008 [36].

On the other hand, other enriched visualization technologies have recently seen widespread adoption in shopping apps that allow customers to visualize and virtually experience products in the course of online shopping. Examples include "Ikea Place," a smartphone app that allows Ikea shoppers to visualize what the company's furniture will look like in their own home, Sephora's "Virtual Artist" that allows customers to visualize make-up products on their own face, and the Gap's "Virtual Dressing Room" that simulates what clothing items would look like on oneself.

Importantly, extant research suggests that visually rich modalities can alter how 
consumers make decisions. For instance, Fritz and colleagues [28] demonstrate that because augmented-reality technologies visually superimpose products into a consumer's real-time environment, these applications can increase the ease of mentally simulating product consumption and in turn increase purchase likelihood. More broadly, media visualization, richness, and interactivity have been shown to boost consumer involvement with products online, which in turn has been found to enhance product learning and satisfaction [82]).

What is less clear, however, is whether engaging in activities through visual modalities changes how consumers think and make decisions relative to interactions on visually impoverished settings (e.g., a traditional retail webpage) or, at the other extreme, during inperson interactions. One early indication that video-based interaction may indeed alter how we think comes from Brucks and Levav [10], who studied how consumers' creativity is affected when dyadic collaborations occur through a video call on a computer (vs. face-to-face). Their central finding is that video-based interactions constricted or limited people's cognitive focus to the screen through which they were interacting, thereby inhibiting divergent thinking and, thus, the number of ideas generated. On the other hand, this same focus on visually mediated interaction allowed video-based dyads to engage in more convergent thinking and thereby better identify the highest-quality ideas. While the authors did not explore whether these effects generalize to other visual interfaces, future work might examine whether interactions with visualization tools such as VR and AR similarly constrict users' focus, and if so, how this might affect decision-making processes.

\section{The Modifiers: How Intelligent Agents Are Altering Choice}

As illustrated in Figure 1, perhaps the most direct way that technology is changing decision-making is through intelligent agents, which can take a variety of forms that differ in the 
degree to which they take over elements of a choice process. Search engines, for example, might be seen as the least invasive, designed to provide the information one searches for in order to make a choice, while leaving the actual choice to the consumer. Smart objects are often at the opposite extreme, at times fully replacing consumers in all phases of decision-making, from information gathering, to information assessment, to the choice itself. In this section, we begin by discussing the effects of interacting with recommendation systems, followed by the consequences of using search agents on consumer search, perceived knowledge, and choice.

\subsection{Recommendation Systems}

Arguably the most well-studied intelligent aids are recommendation systems. These systems are often machine-learning algorithms that attempt to "learn" a consumer's preferences from past choices and then use that information to make suggestions about everything from products consumers should buy to musical artists they should be following [97]. While a number of machine-learning approaches exist for the development of recommendation systems, perhaps the most well-known is collaborative filtering [81], which leverages choice data from multiple users to make predictions about individual-level preferences.

Do recommendation agents enhance consumer welfare? On one hand, there is a body of evidence showing that well-calibrated recommendation technology simultaneously decreases choice effort while increasing choice quality. Work by Häubl and Trifts [35], for example, found that consumers are more likely to be led to welfare-maximizing choices when aided by a product-screening tool calibrated on self-explicated attribute importance weights. Likewise, Dabholkar and Sheng [19] reported that product selections made with the assistance of an agent resulted in high post-choice satisfaction (see also $[80,84,96]$ ). On the other hand, while recommendation systems may offer benevolent outcomes, they carry with them a potential dark 
side: the more consumers delegate their decisions to intelligent agents, the greater the risk that preferences and choices could become misaligned, either unintentionally (e.g., due to imperfections in the automated system) or deliberately (e.g., due to intentional exploitation by firms or other parties).

An example of the latter risk is provided in recent work by Hildebrand and Bergner [37], who studied how investor choices of financial products can be swayed by the format of "robo advice." In one experiment, a sample of customers of an online investment firm were first presented with a series of questions designed to elicit their risk tolerance in investing. In one group the information was elicited in a traditional survey-like format, while in the other it was elicited through a chatbot-based robo advisor interface. Customers were then presented with portfolio recommendations that, while allegedly customized based on their responses, were actually intentionally misaligned with their preferences. The results showed that while the advice was declined by $60 \%$ of participants who interacted with a non-conversational advisor, only $27 \%$ of those interacting with a conversational advisor declined it. This difference arose in part because investors viewed the robo advisor as more human-like, trustworthy, and ultimately benevolent. In short, the machine advisor was able to dupe investors into making non-welfaremaximizing decisions by appearing more human-like.

The extent to which consumers place their trust in recommendation agents is determined in part by how much of the decision-making process is overtaken by the algorithm. One of the earliest demonstrations of this was provided by Dawes [21], who found that even though an algorithm (here, a simple linear model of scores) could predict success in graduate school better than a human admissions committee, people were still unwilling to turn over the role to a model. More recently Dietvorst et al. [23] noted that this could be considered part of a more general 
phenomenon known as "algorithm aversion"-for the most part, given a choice between making a decision by themselves versus turning it over to a computer agent, people prefer doing it themselves [14]. However, as the aforementioned findings of Hildebrand and Bergner [37] suggest, when algorithms simply serve as a source of decision support—offering advice that consumers are free to ignore - this seems to mitigate algorithm aversion $[54,68]$. To illustrate, Dietvorst et al. [24] and Goodwin et al. [30] find that consumers are attracted to algorithm advice when they have insight into the algorithm rather than it being a "black box" that supplants them as a decision maker. In the context of recommendation systems, this allows for the possibility that consumers will gradually migrate from initially considering the advice of recommendation agents to blindly trusting it; the more consumers see that the advice given is reasonable (e.g., Spotify song recommendations), the more trusting they become, which is a critical driver of algorithm acceptance [68].

\subsection{Search Assistants}

While recommendation agents provide a clear example of how algorithms are augmenting choice, even more pervasive has been the impact of search engines, which have revolutionized how consumers gather and interact with external information. Modern search technologies do more than just index links to other websites; they offer direct answers to simple questions, provide personally tailored search results, and even anticipate queries before they are fully formed. Search engines make the ever-growing supply of online information useful by ensuring that this information is not just theoretically available but also easily accessible. Whether it is a hungry consumer looking for restaurant reviews, a student looking for answers to a homework question, or a tourist hoping to get directions, Google is there to provide the answer - effortlessly and instantaneously. 
A growing body of consumer research has begun to explore how on-demand access to external information may transform consumer decision processes — not just by influencing the extent and type of external information consumers consider when making purchase decisions $[55,66]$ but also by more fundamentally blurring the distinction between internal and external knowledge. This more recent research suggests that reliance on the Internet for information shapes both what consumers know and what they think they know [94]. Search engines may undermine objective knowledge, or what people actually know, by encouraging consumers to rely on easily accessible external information rather than developing their own internal knowledge; when people believe information is available and accessible online, they are less likely to remember it themselves [77]. At the same time, search engines may ironically inflate subjective knowledge, or what people think they know; when people use Google to access external information, they often misattribute this information to their own internal knowledge [90]. Taken as a whole, this research suggests that search engines and other technologies offering on-demand access to online information may cause consumers to rely on the Internet as a form of prosthetic memory — one that impedes the development of objective knowledge while simultaneously and ironically inflating subjective knowledge.

In the first demonstration of this "Google Effect" on subjective knowledge, Ward [90, 91] had participants complete a test of general knowledge either with or without Google. Unsurprisingly, those with access to Google performed far better than those who relied only on their own knowledge. More striking, however, is the evidence that participants who used Google erroneously attributed their performance (and the Internet's knowledge) to themselves. Relative to participants who relied on their own knowledge, those who used Google predicted that they would perform significantly better on a second test to be taken without any external help, 
indicating that they did not sufficiently appreciate Google's contributions to the first test.

Moreover, those who used Google reported significantly higher levels of confidence in their own ability to think about and remember information. Subsequent research has revealed that this effect generalizes from misattributions of knowledge for specific information to confidence in one's ability to explain how things work [27], and that simply knowing that the Internet is "remembering" information on one's behalf is sufficient to increase consumer confidence [6].

Further research suggests that the Google Effect on subjective knowledge is quite robust and can influence decisions in important real-world domains. For example, participants in one study were either directly provided with financial information or asked to search Google for it prior to betting on and completing an incentive-compatible investment choice task. Those who used Google bet significantly more on their own performance — and took on significantly more financial risk—yet performed significantly worse [93]. Although artificially inflated confidence can be problematic when there is an objectively optimal choice, it may have more nuanced implications when choice quality is subjective. In another study, participants who Googled information about film-making prior to choosing and watching a film were more confident in their ability to choose the best film, and they reported higher levels of enjoyment after watching — despite the fact that all participants actually watched the same film [31]. In subjective choice contexts, Google-induced confidence may enhance chosen experiences by creating selffulfilling prophecies of positive outcomes.

\section{General Discussion}

Digital innovations are revolutionizing markets by changing how goods and services are made, sold, and consumed [11]. In this paper, we provide an overview of an emerging field of research that examines the more subtle downstream effects of digital innovations: how they are 
altering the ways consumers think, interact, and decide. Our central thesis is that digital innovations have uprooted the traditional relation long assumed to exist between preference and choice. Choices in today's marketplace are often the outcome of a technology-augmented process in which behavior is partially a reflection of the preferences of consumers, but at times also partially a reflection of the medium and modality on which those choices were formed and/or an algorithmic representation of one's preferences provided by intelligent modifiers. Here we attempt to characterize this human-technology interaction and showcase some of what has been learned thus far about its nature and consequences.

One question that might arise is the extent to which these ideas are truly novel. Throughout history, markets have continuously been transformed by innovations in technology, most recently ranging from the rise of telecommunication to increases in the speed and scale at which consumer data can be gathered and processed [18, 25]. However, one factor that makes the current epoch different is that now technical innovations often operate at a truly disaggregate level, providing personalized recommendations that alter how consumers search and decide. As such, the technology tends to be fully embedded in the market demand system. Additionally, consumers are now forming decisions across a variety of new mediums or device types that vary not just in their functionalities but in their interaction modalities, which is serving to further shape the psychological experience of decision-making — and potentially altering the decisions themselves.

These insights hold at least two major implications for marketing research. The first is that it underscores the need to better understand how the physical medium of choice (e.g., wearable technology), the modality of interaction (e.g., touch), and the algorithmic modifier (e.g., smart agents) affect consumer decision-making. Classic marketing research on choice has 
long viewed consumers as autonomous decision makers who, armed with their preferences, make unaided choices of a preferred alternative. As shown in Figure 1, however, this view overlooks the modern reality of choice, where new technology is often a full partner in consumers' choice process. While a rich stream of research has developed over the past few years on the nature of consumer-technology interactions, much work still needs to be done to develop a deeper understanding of these new choice contexts.

The second implication operates at the market scale. A major unknown at this point is whether the rise of technology-augmented decision-making will work to enhance or degrade consumer welfare. On one hand, if technologies such as recommendation systems and smart devices can be assumed to offer an unbiased analog representation of consumer preferences, the effect would be innocuous and presumably welfare-enhancing. In this case, technology would be ensuring a consistently better match between consumer preferences and market alternatives, all at a lower cost of thinking and transacting. However, we provided evidence for how the embedding of technology in a demand system can sometimes work to distort demand - such as when recommendation systems are designed to steer consumers to specific products preferred by the firm, or when consumers' trust in human-like agents is exploited in a way that harms welfare. An important area for future research will be to study such detrimental effects of technologyaugmented choice, along with developing policies that might be enacted to temper them.

In terms of the future of technology-augmented choice, recent years have witnessed enormous advances in technology that affect how we reason, choose, and consume. The technologies highlighted here — ranging from touch-sensitive mobile devices, to voice-controlled digital assistants, to intelligent recommendation agents — offer illustrations of the growing interconnectedness that exists between consumers and technology. We believe that this 
interdependence will only continue to increase as technology becomes more intelligent and facile.

For example, new forms of cross-modal interactions are being generated as we gradually move beyond single-sensory input technologies (such as touch-only or voice-only). Technologies such as Microsoft Kinect, HoloLens, or new features in Google's Android operating system allow for an extensive use of gesture-based control without even touching the object (i.e., the device itself). Likewise, MIT's AlterEgo provides a speak-silent interface that allows humans to interact with machines simply through their neuromuscular signals triggered by subvocalization or internal speech [49]. These recent developments raise fundamental questions about whether the psychological processes discovered in previous research (such as endowment and ownership effects in research related to touch) generalize to new affordances by entirely new classes of human-machine interfaces. These developments also raise questions related to practice and theory development, such as whether these new modalities will alter how consumers search, shop, and express their preferences. We hope that our proposed technology-augmented model of choice will provide a generalizable framework for assessing how different new technologies may alter primitive choice elements (cognitions, emotions, and preferences) and thereby change the choices and behaviors consumers engage in.

Additionally, the technologies discussed in this work produce novel forms of data with the potential to provide a new lens for the study of consumer choice, potentially offering deeper insights into choice processes. These types of data include individual-level sensor data from consumers' mobile devices [89], voice samples during interactions with voice assistants [38], individual-level clickstream data [71], and fine-grained tracking of individual-level choice processes in physical stores [88]. These developments suggest an opportunity for consumer 
researchers to engage in wider-ranging cross-disciplinary research (e.g., collaborating with computer and data scientists) that leverages methodologies outside the boundaries of our own discipline.

In sum, we hope that the research featured in this paper not only inspires new research avenues but also promotes a novel lens to better describe, analyze, and ultimately understand the modern technology-augmented consumer. With the increasing breadth and diversity of humanmachine interactions, we believe there is great potential for marketing researchers to combine and analyze multi-modal data, from voice and biometric data to rich sensor data collected from wearables and mobile devices. 


\section{References}

1. Agrawal A, Gans J, Goldfarb A (2018) Prediction machines: The simple economics of artificial intelligence. Harvard Business Review Press, Boston

2. Alcañiz M, Bigné E, Guixeres J (2019) Virtual reality in marketing: A framework, review, and research agenda. Frontiers in Psychology, 10:1530

3. Ansari A, Essegaier S, Kohli R (2000) Internet recommendation systems. J Mark Res, 37:363375

4. Bettman JR, Luce MF, Payne JW (1998) Constructive consumer choice processes. J Consum Res, 25:187-217

5. Bharadwaj R (2019). Artificial intelligence in home robots: Current and future use-cases. Emerj.com, February 10, 2019. Accessed at: https://emerj.com/ai-sectoroverviews/artificial-intelligence-home-robots-current-future-use-cases/

6. Bhargave R, Mantonakis A, White K (2016) The Cue-of-the-Cloud Effect: When reminders of online information availability increase purchase intentions and choice. J Mark Res, 53:699-711

7. Brasel AS, Gips J (2015) Interface psychology: Touchscreens change attribute importance, decision criteria, and behavior in online choice. Cyberpsychology, Behavior and Social Networking, 18:534-538

8. Braun M, Mainz A, Chadowitz R, Pfleging B, Alt F (2019) At your service: Designing voice assistant personalities to improve automotive user interfaces. In Proceedings of the 2019 CHI Conference on Human Factors in Computing Systems, May:1-11

9. Brown D (2019) Apple watch or it doesn't count: How tech addiction may be ruining your workout. USA Today, May 15, 2019. Accessed at: https://www.usatoday.com/story/tech/2019/05/15/fitbits-apple-watches-and-smartphonescan-actually-hurt-your-workout/1186522001/

10. Brucks M, Levav J (2019) Technology-mediated innovation. Paper presented at Society for Consumer Psychology Boutique: Consumers and Technology, HEC Montreal, Canada

11. Brynjolfsson E, McElheran K (2016) The rapid adoption of data-driven decision-making. American Economic Review, 106:133-139

12. Burke RR (1997) Do you see what I see? The future of virtual shopping. Journal of the Academy of Marketing Science, 25:352-360

13. Burke RR, Harlam BA, Kahn BE, Lodish LM (1992) Comparing dynamic consumer choice in real and computer-simulated environments. J Consum Res, 19:71-78 
14. Burton J, Stein MK, Jensen TB (2019) A systematic review of algorithm aversion in augmented decision making. Journal of Behavioral Decision Making, 3:220-239

15. Cheever N A, Rosen L D, Carrier M, Chavez A (2014) Out of sight is not out of mind: The impact of restricting wireless mobile device use on anxiety levels among low, moderate and high users. Computers in Human Behavior, 37: 290-97

16. Çiçek M (2015) Wearable technologies and its future applications. International Journal of Electrical, Electronics and Data Communication, 3:2320-2084

17. Clayton R B, Leshner G, Almond A (2015) The extended iSelf: The impact of iPhone separation on cognition, emotion, and physiology. Journal of Computer-Mediated Communication, 20: 119-35

18. Cowan RS (1997) A social history of American technology. Oxford University Press, New York

19. Dabholkar P, Sheng X (2012) Consumer participation in using online recommendation agents: Effects on satisfaction, trust, and purchase intentions. The Service Industries Journal, 32:1433-1449

20. Davidson, J (2019) Here's one way to break your smartphone addiction. Financial Review. April 1, 2019. Accessed at: https://www.afr.com/technology/heres-one-way-to-breakyour-smartphone-addiction-20190324-h1cqoy

21. Dawes, RM (1971) A case study of graduate admissions: Application of three principles of human decision making. American Psychologist, 26:180-188

22. Diehl, K, Zauberman G, Barasch A (2016) How taking photos increases enjoyment of experiences. Journal of Personality and Social Psychology, 111:119-140

23. Dietvorst B, Simmons JP, Massey C (2015) Algorithm aversion: People erroneously avoid algorithms after seeing them err. Journal of Experimental Psychology: General, 144:114 126

24. Dietvorst BJ, Simmons, JP, Massey C (2016) Overcoming algorithm aversion: People will use imperfect algorithms if they can (even slightly) modify them. Management Science, 64:1155-1170

25. Dzyabura D, Yoganarasimhan H (2018) Machine learning and marketing, in Handbook of Marketing Analytics: Methods and Applications in Marketing, Public Policy, and Litigation Support, editors Hanssens, D., and Mizik, N.

26. Etkin, J (2016) The hidden cost of personal quantification, J Consum Res, 42:967-984 
27. Fisher M, Goddu MK, Keil FC (2015) Searching for explanations: How the Internet inflates estimates of internal knowledge, Journal of Experimental Psychology: General, 144(3):674

28. Fritz W, Hadi R, Stephen, A (2020) A feast for the eyes: How augmented reality influences food desirability. Working Paper

29. Gartner Research (2019) Gartner Says 100 Million Consumers Will Shop in Augmented Reality Online and In-Store by 2020. Accessed at: https://www.gartner.com/en/newsroom/press-releases/2019-04-01-gartner-says-100million-consumers-will-shop-in-augme

30. Goodwin P, Gönül M, Önkal D (2013) Antecedents and effects of trust in forecasting advice. International Journal of Forecasting, 29(2):354-366

31. Grillo TLH, Pizzutti C, Ward AF (2020) Google-induced decision confidence enhances experiences. Working Paper

32. Hadi R, Venezuela A (2019) Good vibrations: Consumer responses to technology-mediated haptic feedback. J Consum Res, published online. Available at: https://doi.org/10.1093/jcr/ucz039

33. Hadi R, Valenzuela A, Groth OH (2019) A tactile toolbox: Documenting consumer responses to haptically-augmented mobile advertising. Working Paper

34. Hampton W, Hildebrand C. (2019) Good buzz, bad buzz: Using vibrotactile feedback to shape consumer choice. Working Paper

35. Häubl G, Trifts V (2000) Consumer decision making in online shopping environments: The effects of interactive decision aids. Mark Sci, 19:4-21

36. Heath D, Heath C (2011) Why Second Life failed", Slate, November 11, https://slate.com/business/2011/11/why-second-life-failed-how-the-milkshake-test-helpspredict-which-ultrahyped-technology-will-succeed-and-which-wont.html

37. Hildebrand C, Bergner A (2020). Conversational Robo Advisors as Surrogates of Trust: Onboarding Experience, Firm Perception, and Consumer Financial Decision Making, Working Paper

38. Hildebrand C, Efthymiou F, Busquet F (2020) Voice Analytics in Business Research: Conceptual Foundations, Acoustic Feature Extraction, and Applications. Working Paper

39. Hildebrand C and Levav J (2020). Interface haptics and consumer choice. Working Paper

40. Hoffman DH, Novak TP (2018) Consumer and object experience in the internet of things: An assemblage theory approach. J Consum Res, 44:1178-1204 
41. Howell J. (2017) Number of connected IoT devices will surge to 125 billion by 2030, IHS markit says, IHS Markit, October 24, 2017.

42. Hyman IE, Matthew BS, Wise BM, McKenzie KE, Caggiano JM (2010) Did you see the unicycling clown? Inattentional blindness while walking and talking on a cell phone. Applied Cognitive Psychology, 24:597-607

43. Isen A. (2001) An influence of positive affect on decision making in complex situations: Theoretical issues with practical implication. J Consum Psych, 11:75-85

44. Jakicic JM, Davis KK, Rogers R, King WC, Marcus MD, Helsel D, Rickman AD, Wahed AS, Belle SH (2016) Effect of wearable technology combined with a lifestyle intervention on long-term weight loss: The IDEA randomized clinical trial. Journal of the American Medical Association, 316:1161-1171

45. Jeng W, He D, Jiang J (2013) Users' perceived difficulties and corresponding reformulation strategies in voice search. The 7th Annual Symposium on Human-Computer Interaction and Information Retrieval

46. Jiang J, Jeng W, He D (2013) How do users respond to voice input errors? Lexical and phonetic query reformulation in voice search. Proceedings of the 36th International ACM SIGIR Conference on Research and Development in Information Retrieval, 143152

47. Kahn BE, Isen A. (1993) The influence of positive affect on variety seeking among safe, enjoyable products. J Cons Res, 20:257-270

48. Kaplan AM, Haenlein M (2009) Consumer use and business potential of virtual worlds: The case of "Second Life." International Journal of Media Management, 11:93-101

49. Kapur A, Kapur, S, Maes P (2018, March) Alterego: A personalized wearable silent speech interface. In 23rd International Conference on Intelligent User Interfaces, 43-53

50. Klesse A K, Levav J, Goukens C (2015) The effect of preference expression modalities on self-control. J Consum Res, 42: 535-50

51. Kushlev K, Proulx JDE, Dunn EW (2016) Silence your phones: Smartphone notifications increase inattention and hyperactivity symptoms. Proceedings of CHI' 16

52. Legault L (2016) Intrinsic and extrinsic motivation. Encyclopedia of Personality and Individual Differences, 10

53. Lin MB, Huang Y (2017) The impact of walking while using a smartphone on pedestrians' awareness of roadside events. Accident Analysis and Prevention, 101 (April):87-96 
54. Logg J, Minson J, Moore D (2019) Algorithm appreciation: People prefer algorithmic to human judgment. Organ Behav Hum Decis Process, 151:90-103

55. Lynch Jr. JG, Ariely D (2000) Wine online: Search costs affect competition on price, quality, and distribution, Mark Sci, 19:83-103

56. McFadden D (1974) Conditional logit analysis of qualitative choice behavior, in P. Zarembka, ed., Frontiers in Econometrics, Academic Press, New York, pp. 105-142

57. Melumad S, Inman JJ, Pham MT (2019) Selectively emotional: How smartphone use changes user-generated content. J Mark Res, 56:259-275

58. Melumad S, Meyer RJ (2020) Full disclosure: How smartphones enhance consumer selfdisclosure. J Mark, 84:28-45.

59. Melumad S, Pham MT (2020) The smartphone as a pacifying technology, J Consum Res, published online. Available at: https://doi.org/10.1093/jcr/ucaa005

60. Meyer RJ, Broad K, Orlove B, Petrovic N (2013) Dynamic lab simulation as an approach to understanding hurricane risk response: Insights from the Stormview Lab, Risk Analysis, 33(8):1532-1552

61. Mittelstadt BD, Patrick A, Taddeo M, Wachter S, Floridi L (2016) The ethics of algorithms: Mapping the debate. Big Data \& Society, December

62. Mogilner C, Aaker J, Kamvar SD (2012) How happiness affects choice. J Consum Res, 39(2):429-443

63. Mulcahy R, Riedel AS (2020) Touch it, swipe it, shake it: Does the emergence of haptic touch in mobile retailing advertising improve its effectiveness? Journal of Retailing and Consumer Services, 54

64. Panova T, Carbonell X (2018). Is smartphone addiction really an addiction? Journal of Behavioral Addictions, 7(2):252-259

65. Peck J, Wiggins J (2006) It just feels good: consumers' affective response to touch and its influence on persuasion. J Mark, 70(October):56-69

66. Peterson RA, Merino, MC (2003) Consumer information search behavior and the internet, Psychology and Marketing, 20(2):99-121

67. Pew Research Center (2019) Mobile fact sheet, Report, Pew Research Center, Washington, DC 
68. Prahl A, Van Swol L (2017) Understanding algorithm aversion: When is advice from automation discounted? Journal of Forecasting, 36:691-702

69. Radin JM, Wineinger NW, Topol EJ, Steinhubl SR (2020) Harnessing wearable device data to improve state-level real-time surveillance of influenza-like illness in the USA: A population-based study. The Lancet, e85-e93

70. Ransbotham S, Lurie NH, Liu H (2019) Creation and consumption of mobile word of mouth: How are mobile reviews different? Mark Sci, 38(5), 773-792

71. Rutz OJ. Bucklin RE (2012) Does banner advertising affect browsing for brands? Clickstream choice model says yes, for some. Quantitative Marketing and Economics, 10(2):231-257

72. Sagarin BJ, Britt A, Heider JD, Wood SE, Lynch JE (2003) Bartering our attention: The distraction and persuasion effects of online advertisements. Cognitive Technology, 8:4 17

73. Schirg F, Vandor P (2007) The potential of 'Information Acceleration' for forecasting critical parameters in the space tourism market. Proceedings of the 58th International Astronautical Congress

74. Shelstad W, Smith D, Chaparro B (2017) Gaming on the rift: How virtual reality affects game user satisfaction. Proceedings of the Human Factors and Ergonomics Society Annual Meeting, 61:2072-2076

75. Shen H, Zhang M, Krishna A (2016) Computer interfaces and the "direct-touch" effect: Can iPads increase the choice of hedonic food? J Mark Res, 53(5):745-758

76. Siquid M, Lanker T, Makhdoomi K (2017) Augmented reality v. virtual reality. International Journal of Computers Science and Mobile Computing, 6:324-327

77. Sparrow B, Liu J, Wegner DM (2011) Google effects on memory: Cognitive consequences of having information at our fingertips. Science, 333:776-778

78. Stanton SJ, Reeck C, Huettel SA, LaBar KS (2014) Effects of induced moods on economic choices. Judgment and Decision Making, 9(2):167-175

79. Stothart C, Mitchum A, Yehnert C (2015) The attentional cost of receiving a cell phone notification. Journal of Experimental Psychology: Human Perception and Performance, 41(4):893

80. Su HJ, Comer LB, Lee S (2008) The effect of expertise on consumers' satisfaction with the use of interactive recommendation agents. Psychology and Marketing, 25 (9):859-880 
81. Su X, Khoshgoftaar TM (2009) A survey of collaborative filtering techniques. Advances in Artificial Intelligence

82. Suh KS, Chang S (2006) User interfaces and consumer perceptions of online stores: The role of telepresence. Behaviour and IT, 25:99-113

83. Suh KS, Lee YE (2005) The effects of virtual reality on consumer learning: An empirical investigation. MIS Quarterly, 29(4):673-697

84. Swearingen K, Sinha R (2001) Beyond algorithms: An HCI perspective on recommender systems. Proc. SIGIR 2001 Workshop on Recommender Systems

85. Taylor, P (2009) Text-to-speech synthesis. Cambridge University Press, Cambridge

86. Tversky A, Simonson I (1993) Context-dependent preferences. Management Science, 39:1179-1189

87. Urban GL, Hauser JR, Qualls WJ, Weinberg BD, Bohlmann JD, Chicos, RA (1997)

Information acceleration: Validation and lessons from the field. J Mark Res, 34:143-153

88. Walter M, Hildebrand C, Häubl G, Herrmann A (2020) Mixing it up: Unsystematic product arrangements promote the choice of unfamiliar products. J Mark Res, published online. Available at: https://doi.org/10.1177/0022243720901520

89. Wang W, Harari GM, Wang R, Müller SR, Mirjafari S, Masaba K, Campbell AT (2018) Sensing behavioral change over time: Using within-person variability features from mobile sensing to predict personality traits. Proceedings of the ACM on Interactive, Mobile, Wearable and Ubiquitous Technologies, 2(3):1-21

90. Ward AF (2013a) One with the cloud: Why people mistake the internet's knowledge for their own. Doctoral Dissertation, Harvard University

91. Ward AF (2013b) Supernormal: How the internet is changing our memories and our minds. Psychological Inquiry, 24(4):341-348

92. Ward AF, Duke K, Gneezy A, Bos M (2017) Brain drain: The mere presence of one's own smartphone reduces available cognitive capacity. Journal of the Association for Consumer Research, 2(2):140-154

93. Ward AF, Grillo TLH, Fernbach, PM (2020). Risky click: Online financial search and consumer financial decision-making. Working Paper

94. Wegner DM, Ward AF (2013) How Google is changing your brain. Scientific American, 309(6):58-61 
95. Williams R (2019) Visa's sensory branding boosts positive perception by $14 \%$. Marketing Dive. Accessed at: https://www.marketingdive.com/news/visas-sensory-branding-drives14-gain-in-positive-brand-perception/557235/.

96. Wu L, Joung Y, Lee J (2013) Recommendation systems and consumer satisfaction online: Moderating effects of consumer product awareness. Proceedings of the Annual Hawaii International Conference on System Sciences, 2753-2762

97. Xiao BS, Benbasat I (2007) E-commerce product recommendation agents: Use, characteristics, and impact. MIS Quarterly, 31:137-209

98. Xu J, Ding M (2019) Using the double transparency of autonomous vehicles to increase fairness and social welfare. Cust Needs Solut, 6:23-35 